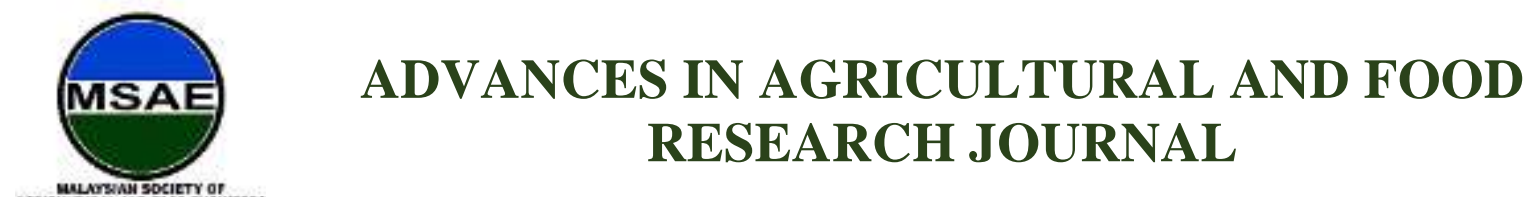

Original Research Article

\title{
Effect of Different Jackfruit Puree Concentrations on the Mechanical Properties of Jackfruit Frozen Confection
}

\author{
Amiruddin Mat Johari ${ }^{1}$, Nur Aliaa Abd Rahman ${ }^{1 *}$, Roseliza Kadir Basha ${ }^{1}$, Azhari Samsu Baharuddin ${ }^{1}$, Mohd \\ Afandi P. Mohammed ${ }^{1}$, Ahmad Tarmezee Talib ${ }^{1}$, Minato Wakisaka ${ }^{2}$ \\ ${ }^{1}$ Department of Process and Food Engineering, Faculty of Engineering, Universiti Putra Malaysia, 43400 \\ UPM Serdang, Selangor \\ ${ }^{2}$ Graduate School of Life Science and Systems Engineering, Kyushu Institute of Technology, 2-4 Hibikino, \\ Wakamatsu-ku, Kitakyushu, 808-0196, Japan \\ *Corresponding author: Nur Aliaa Abd Rahman, Department of Process and Food Engineering, Faculty of \\ Engineering, Universiti Putra Malaysia, 43400 UPM Serdang, Selangor, Malaysia; nuraliaa@upm.edu.my
}

\begin{abstract}
Jackfruit frozen confection has been mechanically characterised in situ by using compression tests. There are no available studies on the mechanical behaviour of jackfruit frozen confection. The aim of this study is to identify the mechanical properties of jackfruit frozen confections formulated with different concentrations of jackfruit puree. In this study, the experimental analyses are conducted using a compression test device made from LEGO Mindstorms EV3. The portable device is placed inside a freezer to enable the measurements to be done in low temperatures $\left(-20^{\circ} \mathrm{C}\right)$. This is to overcome the limitation of an actual texture analyser which can only be operated at room temperature. The mechanical properties of jackfruit frozen confections at different jackfruit puree concentrations (10\%, $20 \%$ and $30 \%$ ) are obtained using the tester and analysed. The tests conducted are uniaxial compression, stress relaxation test and multi-step stress relaxation test. It has been observed that frozen confection with $20 \%$ jackfruit puree concentration (JF20) is able to withstand a higher force of compression $(27.79 \mathrm{kPa})$ compared to the ones with $10 \%$ (JF10) and $30 \%$ (JF30) concentrations, at $21.15 \mathrm{kPa}$ and $10.48 \mathrm{kPa}$, respectively. For stress relaxation test, JF30 has the highest increasing stress for a strain of 0.05 to 0.2 but it decreases at a strain of 0.3 to 0.4 . The results of the multi-step relaxation test on JF30 show agreement with the other two tests where the stress decays starting from the $3^{\text {rd }}$ step until the $5^{\text {th }}$ step of the test. This study provides information on the behaviour of jackfruit frozen confection when subjected to compression and stress that imitates the movement during consumption.
\end{abstract}

Keywords: Mechanical characterisation; jackfruit; frozen confection; dairy processing

Received: $28^{\text {th }}$ June 2020

Citation: Mat Johari A, Abd Rahman NA, Kadir Basha R, et al. Effect of different jackfruit puree concentrations on the mechanical

Accepted: $10^{\text {th }}$ September 2020 properties of jackfruit frozen confection. Adv

Published: $30^{\text {th }}$ September 2020 Agri Food Res J 2020; 1(1): a0000110. https://doi.org/10.36877/aafrj.a0000110 


\section{Introduction}

Ice cream refers to frozen creamy dessert with a fat content of more than $10 \%$ while frozen confection contains fat of less than $10 \%$ (Johari et al., 2020). In this study, a study on jackfruit frozen confection is conducted. The complex physical structure of frozen confection presents challenges to the producers during production, distribution and consumption. Specifically, jackfruit frozen confection properties need to be properly studied to solve these challenges. Jackfruit is the main ingredient in frozen confection as it influences the overall flavour. Therefore, its addition would also play a role in the structure of frozen confection. The shelf life of jackfruit may affect the texture and the physico-chemical properties of frozen confection such as the mix viscosity, melting characteristics and overrun. The fruit concentration may also affect the mechanical behaviour of frozen confection such as its viscosity, compressive strength, hardness and stress relaxation. However, certain experiments need to be performed under subfreezing temperature as frozen confection in general, is sensitive to temperature fluctuation just like ice cream. This prevents frozen confection from deforming due to melting. A change in temperature could affect the physical and chemical properties of ice cream (Leducq et al., 2015). The same issue that would affect frozen confection too.

Just like ice cream, the frozen confection is a temperature-sensitive product. Temperature fluctuation during sample preparation and experimental work could change the physical and chemical properties of ice cream (Leducq et al., 2015). Performing mechanical tests on frozen confection would require a controlled environment and proper laboratory instruments. The main limitation is due to the temperature-sensitive properties of frozen confection which require experimental procedures performed at sub-freezing temperature, i.e., -10 to $-18^{\circ} \mathrm{C}$. This is to prevent frozen confection from deforming due to melting. Thus, a compression tester was designed to fit into a controlled temperature freezer. By using the miniature compression tester from LEGO Mindstorms EV3 (Rahman et. al., 2019), mechanical tests could be performed in the limited space freezer. LEGO Mindstorms EV3 consists of microprocessors, motors and sensors that could be programmed to perform complicated tasks. MATLAB programming was used to navigate and synchronise the movements of the plate, sensors and the load cell to obtain data easily and systematically.

Mechanical loading tests differ according to the sample tested. The types of tests that are suitable to be used for frozen confection are the uniaxial compression, stress relaxation and cyclic compression. A compression test is important as it provides analytical insights on the properties of ice cream/frozen confection. It stimulates the forces imparted on the product 
during eating, and also in extrusion and packaging of ice cream during its processing stage. Research showed that a strong compressive strength was needed for ice cream with high milk fat percentage (Casarotto et al., 2015). In order to achieve true uniaxial stress without shear deformation, the platen interface and samples need to be properly staged. The inability to do so will produce friction between the sample and loading platen interface which causes inhomogeneous uniaxial stress. This effect is also known as the "friction hill" that causes the barrelling of the sample. A cylindrical shape sample through compression may exhibit this phenomenon whereby the diameter half through the length of the compressed sample is larger than the diameter at the top and bottom edges (Çetinarslan, 2007).

Stress relaxation is a time-dependent process that allows a material to slowly relax at a constant strain that leads to a decrease in stress performed on the sample. This test provides valuable information specifically on the rheological parameters of viscoelastic foods (Heldman et al., 2006). Ideal viscous substances relax instantaneously while ideal elastic materials show no relaxation. Viscoelastic materials relax gradually and stop depending on the molecular structure of the material. Stress in viscoelastic solids would decay to equilibrium stress that is greater than zero, but residual stress in viscoelastic liquids would decrease to zero (Banes, 2016). In the stress relaxation of the ice cream/confection sample, the stress will decay as it remains at a certain strain. Therefore, proper relaxation time is important as it influences the stress-strain measurements. The aim of this study is to identify the mechanical properties of jackfruit frozen confections formulated with different concentrations of jackfruit puree.

\section{Materials and Methods}

\subsection{Preparation of Jackfruit Frozen Confection}

The base formulation of frozen confection had the following composition (\%w/w): $29.1 \%$ water, $46.5 \%$ full cream milk, $16.5 \%$ sugar, $3.6 \%$ whey powder, $3.6 \%$ creamer, $0.4 \%$ emulsifier and $0.3 \%$ stabiliser. This was based on the formulation used by Rahman et al. (2019) with some modifications. The wet and dry ingredients were thoroughly agitated by manual mixing at room temperature. The mix was batch pasteurised at $80^{\circ} \mathrm{C}$ for 15 seconds and later homogenised using a laboratory-scale homogeniser. The liquid mixes were then aged overnight at $0-5^{\circ} \mathrm{C}$. Jackfruit puree was incorporated into the aged mixes as suggested by Goff and Hartel (2013), and the jackfruit frozen confection mixes were then frozen using an ice cream machine (Model BCI600XL, Breville, USA). The produced frozen confections 
were stored in a deep freezer at $-18^{\circ} \mathrm{C}$. Based on Goff and Hartel (2013), the recommendation of fresh and frozen fruit required to impart the desired flavour varies from 10 to $25 \%$ of the mass of the finished product. Therefore, the jackfruit puree added was 10\% (JF10), 20\% (JF20) and 30\% (JF30) of the total mass of the mix in this experiment.

\subsection{The Setup of LEGO Mechanical Testing System}

The mechanical testing system was comprised of a custom-built LEGO Mindstorms EV3 Robotic Kit, load cell (FUTEK LSB200, USA) and an endoscope (Rahman et al., 2019). The robotic kit was designed with a microcontroller programmed through a USB connection. The structure of LEGO was built with pegs that allows modification when needed as shown in Figure 1. Load cell could operate at sub-freezing temperature $\left(-20^{\circ} \mathrm{C}\right)$ which is suitable with the low-temperature condition needed in frozen confection mechanical testing. The endoscope was used to record and obtain live feeds of the tests. The movement of the LEGO compression tester was set accordingly to specific tests i.e. the uniaxial compression and stress relaxation.

The motor and load cell were controlled by written programming scripts (MATLAB 2014a, USA) to synchronise the movement of the actuator and data acceptance from the load cell. The compression tester was made portable with its small dimension (L: $17.2 \mathrm{~cm}, \mathrm{~W}$ : $14.8 \mathrm{~cm}, \mathrm{H}: 31.2 \mathrm{~cm}$ ). The small size of the compression tester allows convenient placement inside the freezer along with the ice cream samples. A portable compression tester is also important so that it can be moved in and out of the freezer easily. This ensures minimum exposure of the machine to sub-freezing temperature especially during the gaps between testing and loading of the sample. Several challenges were encountered throughout the testing phase. These challenges were the preparation of frozen confection sample, development of the LEGO compression tester and the temperature conditioning of experiments. 


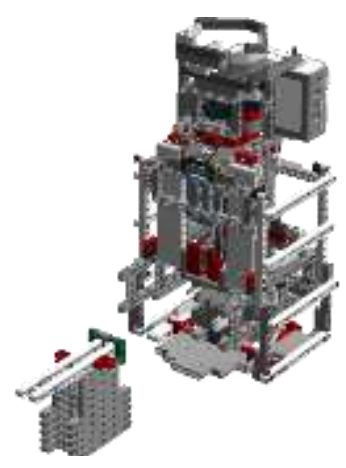

(a)

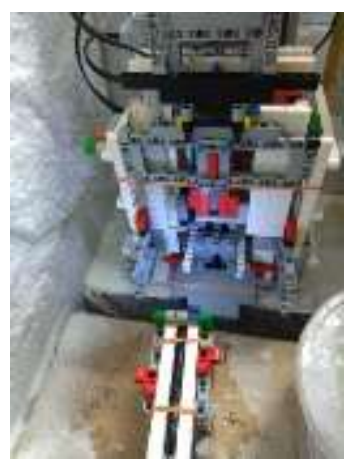

(c)

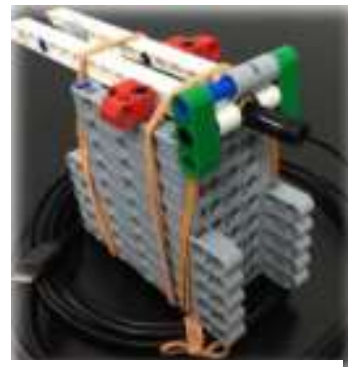

(e)

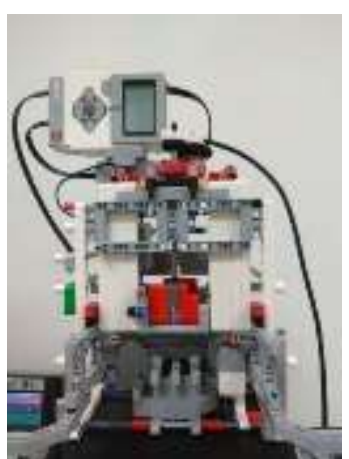

(b)

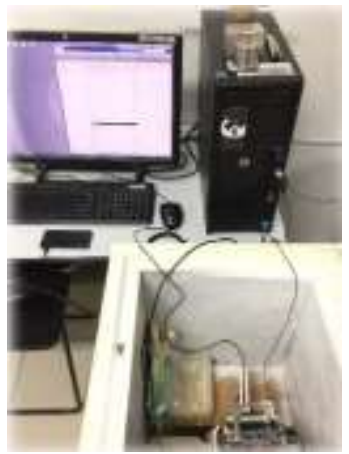

(d)

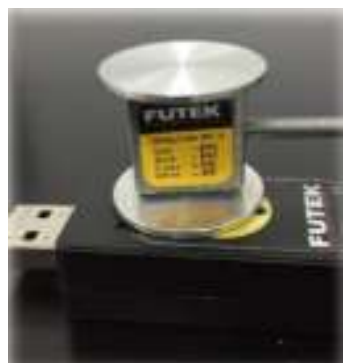

(f)

Figure 1. LEGO Mindstorms EV3 compression tester; (a) LEGO drawing of prototype, (b) Front image of prototype, (c) Prototype in freezer, (d) Wiring of prototype attached to CPU, (e) Endoscope mounted on LEGO holder, (f) FUTEK load cell.

\subsection{Frozen Confection Sample for Mechanical Testing}

The frozen confection samples were produced from the formulation explained previously. Jackfruit puree concentrations were added at $10 \%, 20 \%$ and $30 \%$ of the total mass of liquid mix. After the dynamic freezing of jackfruit frozen confection, the samples were then piped into a cylindrical plastic container $(\mathrm{d}: 1.5 \mathrm{~cm})$. The frozen confections were hardened overnight. After that, the frozen confection samples were cut to a height of $0.75 \mathrm{~cm}$ to produce tablet shape samples (Figure 2). The cutting of samples was done in a freezer to prevent melting. 


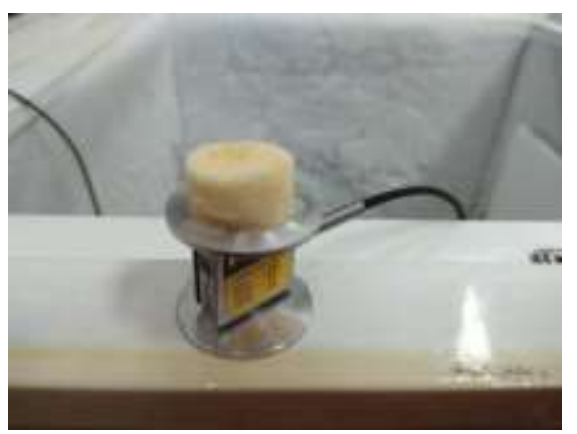

Figure 2. Frozen confection sample with a diameter of $1.5 \mathrm{~cm}$ and height of $0.75 \mathrm{~cm}$.

\subsection{Compression Tests on Jackfruit Frozen Confection}

Compression tests were performed to determine the mechanical properties of jackfruit frozen confection under subfreezing temperature. This prevents further melting of frozen confection which could affect the tests conducted. The compression tests were conducted using a compression tester (LEGO Mindstorms kit) equipped with a load cell (FUTEK LSB200, USA). The tests initiated were uniaxial compression, stress relaxation and stepstress relaxation. The tests were adapted according to Mohammed (2012) with simplifications and performed under sub-freezing temperature and low space of the experimental area. Jackfruit frozen confection samples were transferred from the storage freezer $\left(-18^{\circ} \mathrm{C}\right)$ to a controllable freezer set to $-10^{\circ} \mathrm{C}$. The compression tester and load cell were placed in the controllable freezer 10 minutes earlier before the experiment started. The sample was then placed onto the load cell.

\section{i. Uniaxial compression test}

The uniaxial compression test provides true uniaxial stress without any shear deformation taking place. Jackfruit frozen confection sample was compressed at a speed of $0.2 \mathrm{~mm} / \mathrm{s}$ and a strain of $0.4(3 \mathrm{~mm})$. The stress and strain were computed from the load and displacement obtained. The experiments were performed in triplicate.

ii. Stress and multi-step stress relaxation test

The stress relaxation test was done to investigate the time-dependent behaviour of jackfruit frozen confection. The speed was set at $0.2 \mathrm{~mm} / \mathrm{s}$ and strain of $0.4(3 \mathrm{~mm})$. Relaxation time was set to 30 seconds. Step-stress relaxation test was also conducted with a 5-step compression at a speed of $0.2 \mathrm{~mm} / \mathrm{s}$ and strain of $1 \mathrm{~mm}$. The relaxation time was five seconds in between steps. The experiments were performed in triplicate.

The data of all mechanical tests were analysed in terms of stress $(\mathrm{kPa})$ and strain. The stress is defined as the applied force divided by the original cross-sectional area of the sample. It is 
calculated using equation 1 :

$$
\sigma_{e}=\frac{P}{A_{0}}
$$

where $\sigma_{e}$ is the stress, $P$ is the applied load and $A_{0}$ is the initial cross-sectional sample normal to the loading direction. The strain expresses the length of the deformed sample divided by the initial height of the sample. It is calculated based on equation 2 :

$$
\varepsilon_{e}=\frac{\Delta L}{L_{0}}
$$

where $\varepsilon_{e}$ is the strain, $\Delta L$ is the measured displacement and $L_{0}$ is the initial sample length along a single axis.

\section{Results and Discussions}

\subsection{Mechanical Tests on Jackfruit Frozen Confection at Different Jackfruit Puree Concentrations Using LEGO Mindstorms Compression Tester}

Compression tests on jackfruit frozen confection were performed to obtain the ideal jackfruit puree concentration needed in the sample as a flavouring. Ideal, in this case, means that the frozen confection produced from the puree has an acceptable texture and taste. Mechanical tests were conducted in a controlled temperature freezer using a compression tester made from LEGO Mindstorms kit (EV3 Core Set). This prevented the melting of jackfruit frozen confection which could affect its mechanical properties.

\subsection{Uniaxial Compression Test on Jackfruit Frozen Confection}

The compressive strength of frozen confection indicates significant importance to manufacturers as it relates to handling and extruding of the product. Besides that, the compressive strength also affects consumer preference in terms of its textural characteristics. Uniaxial compression tests were carried out on three different jackfruit puree concentrations. The concentrations were $10 \%, 20 \%$ and $30 \%$ of the total mass of liquid mix. Different concentrations were used to determine the ideal jackfruit puree needed based on the mechanical properties of jackfruit frozen confection. Figure 3 shows the deformation of jackfruit frozen confection at different puree concentrations that were penetrated to a 0.4 (3 $\mathrm{mm}$ ) strain at $0.2 \mathrm{~mm} / \mathrm{s}$. 


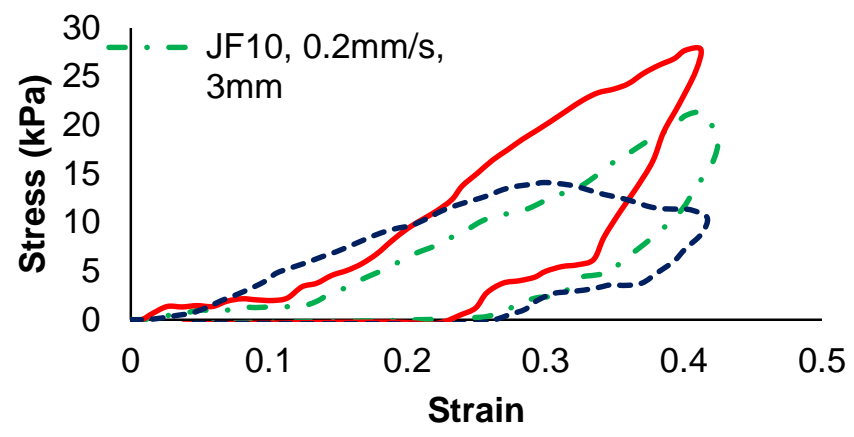

Figure 3. Uniaxial compression test result of jackfruit frozen confection at different jackfruit concentrations.

The graph shows the effect of increasing stress for all three types of jackfruit frozen confections. As the speed increased, the slope of stress versus time increased. At 0.4 (3 mm) strain, JF10 produced a stress of $21.15 \mathrm{kPa}$, JF20 had the stress of $27.79 \mathrm{kPa}$ while JF30 with the stress of $10.48 \mathrm{kPa}$. JF20 had higher stress compared to JF10 due to the effect of jackfruit puree concentration. Jackfruit bulb contains carbohydrates, protein, minerals and fibres (Swami et al., 2012), therefore it would affect the mechanical structure of the frozen confection. In terms of sugar concentration from the fruit puree, the effects were contrasted to the findings of Guinard et al. (1997) which stated that high sugar causes low hardness of frozen confection. Sugar content coming from jackfruit bulbs may not be the main factor in affecting the mechanical properties of jackfruit frozen confection as it opposes the finding stated beforehand. However, water content increases as a higher amount of jackfruit puree was added. Hartel (1996) stated that increasing water in ice cream increases its hardness. Higher water content could also dilute the sugar content provided by the jackfruit puree. Besides that, fibre content reduces the whipping capacity of the ice cream mix. This reduces the overrun of ice cream which subsequently produces harder ice cream. Carbohydrate-based fat replacers had also shown to prevent air incorporation as reported by Adapa et al. (2000).

JF30 had the highest increasing stress for a strain of 0.05 to 0.2 but it decreased at a strain of 0.3 to 0.4 . The high increasing amount of stress in the early stage of compression may be due to the amount of jackfruit moisture content of around 70 to $80 \%$. This contributes more water to the frozen confection as jackfruit puree concentration increases. Increased water would produce larger ice crystals and this will subsequently increase the hardness of ice confection as also stated by Hartel (1996). However, the decrease of stress at a strain of 0.3 to 0.4 is due to the solid-like structure of frozen confection that breaks at certain strains. 


\subsection{Stress-Relaxation Test on Jackfruit Frozen Confection}

Stress relaxation tests were conducted in order to illustrate the viscoelasticity of frozen confection. Results of jackfruit frozen confection at different jackfruit concentrations are shown in Figure 4.

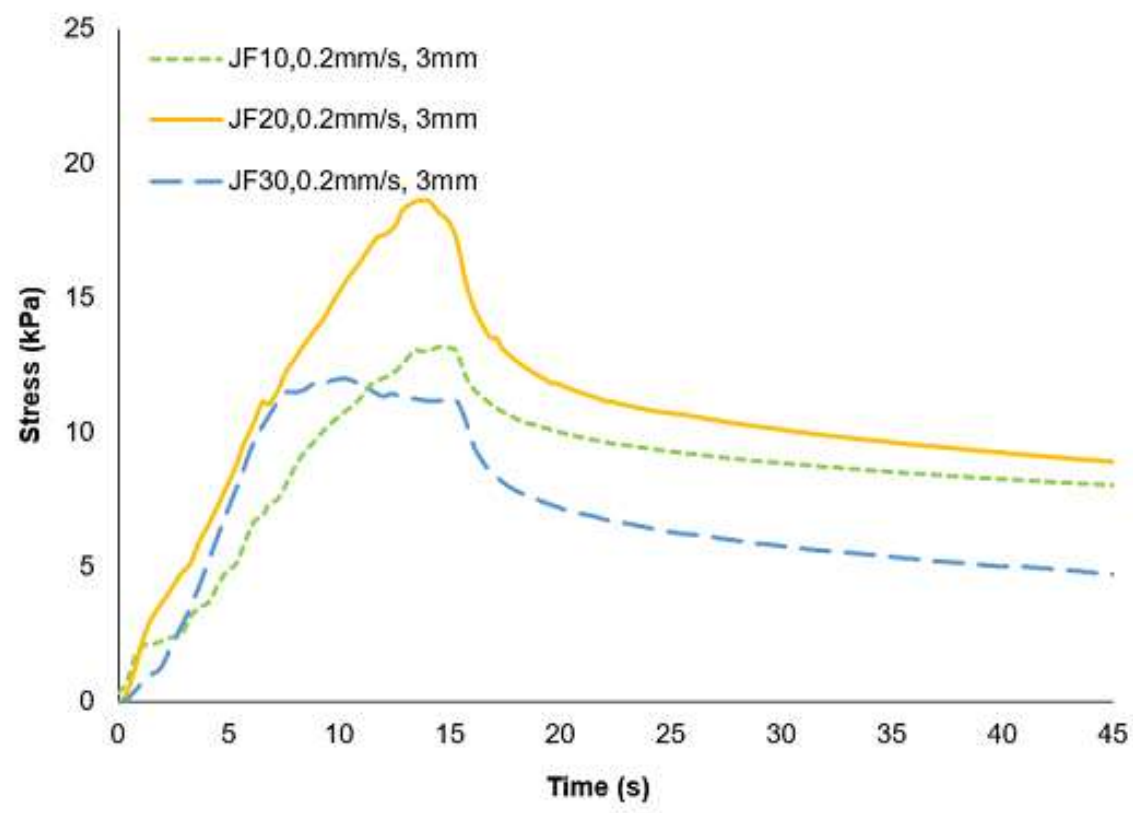

Figure 4. Relaxation test result of jackfruit frozen confection at different jackfruit concentrations.

The jackfruit frozen confection showed viscoelasticity characteristics during the tests. JF10 had increasing stress until 15 seconds of compression with peak stress of less than 15 $\mathrm{kPa}$. A similar trend was seen for JF20 that showed a higher peak of the stress of more than $15 \mathrm{kPa}$. A higher amount of jackfruit concentration increases stress during compression. This is similar to the results obtained for uniaxial compression tests of JF10 and JF20. The effect of stress was likely due to the increase in water content which hardens the frozen confection. However, JF30 exhibited a plateau characteristic from five seconds to 15 seconds of compression. This could explain why JF30 exhibited lesser elastic properties compared to JF10 and JF20. The higher amount of fibre, sugar and pectin from the jackfruit pulp increases the stiffness of JF30 frozen confection. Thus, this solid-like structure would break faster upon reaching a certain strain. The addition of jackfruit puree affects the overrun and consequently hardness of jackfruit frozen confection.

\subsection{Multi-step relaxation test on jackfruit frozen confection}

A multi-step stress relaxation graph is presented in Figure 5. The 5-step relaxation was applied to each sample. It was observed that JF10 graph trend was lower than JF20. Similar 
trends were also observed in the uniaxial compression test and stress relaxation test. The graph for JF30 followed similar curves as JF10 and JF20 up to its third step which was at its $0.4(3 \mathrm{~mm})$ strain. The highest amount of fibre, pectin and sugar in the sample increased the stiffness of JF30, thus showing a solid-like structure and broke at an earlier compression time and strain. This was presented with the dropping decay of stress starting from the third step until the fifth step of the test.

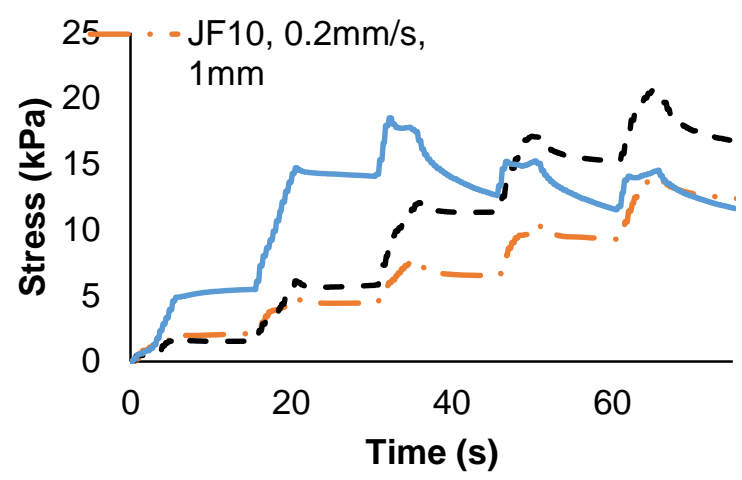

Figure 5. Multi-step stress relaxation test result of jackfruit frozen confection at different jackfruit concentrations.

\section{Conclusion}

Mechanical tests were conducted in a controlled temperature freezer using a compression tester made from LEGO Mindstorms kit (EV3 Core Set). For the uniaxial compression test, JF20 had higher stress compared to JF10 due to the effect of jackfruit puree concentration. For stress relaxation test, JF30 had the highest increasing stress for a strain of 0.05 to 0.2 but it decreased at a strain of 0.3 to 0.4 . JF30 exhibited lesser elastic properties compared to JF10 and JF20. The higher amount of fibre, sugar and pectin from the jackfruit pulp increases the stiffness of JF30 frozen confection. The results of the multi-step relaxation test on JF30 showed agreement with the other two tests where the stress decayed starting from the third step until the final step of the test. JF30 had a solid-like structure and broke at an earlier compression time and strain. Based on the results, it can be concluded that jackfruit frozen confection with $20 \%$ jackfruit puree concentration (JF20) had the desired texture compared to JF10 and JF30 samples.

Acknowledgements: The authors would like to thank Universiti Putra Malaysia (GP/IPS/9518100) for the funding and Pahang State Farmers Association (PASFA) for working closely with us to make this study a success.

Conflicts of Interest: The authors declare that there is no conflict of interest in this work.

\section{References}

Adapa, S., Dingeldein, H., Schmidt K.A. et al., (2000). Rheological properties of ice cream mixes and frozen ice creams containing fat and fat replacers. Journal of Dairy Science, 83(10), 2224-2229. 
Banes, P., (2016). Viscoelastic behavior. European Medical Alliance.

Casarotto, A.M., Wolfang, E. \& Lundgren, K.J. (2015). Effects of composition and flavor on viscoelastic properties of ice cream. In Department of Mechanical Engineering. Worcester Polytechnic Institute.

Çetinarslan, C.S. (2007). Effect of aspect ratio on barreling contour and variation of total surface area during upsetting of cylindrical specimen. Materials \& Design, 28(6), 1907-1913.

Goff, H.D. \& Hartel, R.W. (2013). Ice cream (7th ed.). US: Springer.

Guinard, J.-X., Zoumas-Morse, C., Mori, L., et al. (1997). Sugar and fat effects on sensory properties of ice cream. Journal of Food Science, 62(5), 1087-1094.

Hartel, R.W. (1996). Ice crystallization during the manufacture of ice cream. Trends in Food Science \& Technology, 7(10), $315-321$.

Heldman, D.R., Lund, D.B. \& Sabliov, C. (2006). Handbook of food engineering (2nd ed.). CRC Press.

Johari, A.M., Rahman, N.A.A., Baharuddin, A.S., et al., (2020). Effects of low temperature storage of Mastura (J37) jackfruit bulbs on the physical quality of jackfruit frozen confection. Journal of Agricultural and Food Engineering, $1,1-6$.

Leducq, D., Ndoye, F.T., Charriau, C., et al., (2015). Thermal protection of ice cream during storage and transportation. 24ième Congrès International du Froid ICR 2015.

Mohammed, M.A.P. (2012). Mechanical characterisation, processing and microstructure of wheat flour dough [Doctoral dissertation, Imperial Collage London]. Mechanical Engineering, Imperial College London.

Rahman, N.A.A, Parid, D.M., Abdul Razak, S.Z., et al., (2019). In-situ viscoelastic characterization and modelling of ice cream. Journal of Food Engineering, 263, 96-101.

Swami, S.B., Thakor, N.J., Haldankar, P.M., et al., (2012). Jackfruit and its many functional components as related to human health: A review. Comprehensive Reviews in Food Science and Food Safety, 11(6), 565-576.

Copyright (C) 2020 by Mat Johari A, et al. and HH Publisher. This work is licenced under the Creative Commons

Attribution-NonCommercial 4.0 International Licence (CC-BY-NC4.0) 